OPEN ACCESS

Edited by:

André Ricardo Araújo Lima, Center for Marine and Environmental

Sciences (MARE), Portugal

Reviewed by:

Guilherme Batista Ferreira,

Federal Rural University

of Pernambuco, Brazil

Sebastien Cambier,

Luxembourg Institute of Science

and Technology, Luxembourg

${ }^{*}$ Correspondence: Rafael Trevisan

rafael.trevisan@duke.edu; rafael.trevisan@gmail.com

Specialty section: This article was submitted to Toxicology, Pollution and the Environment,

a section of the journal

Frontiers in Environmental Science

Received: 04 March 2020

Accepted: 19 May 2020

Published: 24 July 2020

Citation:

Trevisan R, Uzochukwu D and Di Giulio RT (2020) PAH Sorption to Nanoplastics and the Trojan Horse Effect as Drivers of Mitochondrial

Toxicity and PAH Localization in Zebrafish. Front. Environ. Sci. 8:78. doi: 10.3389/fenvs.2020.00078

\section{PAH Sorption to Nanoplastics and the Trojan Horse Effect as Drivers of Mitochondrial Toxicity and PAH Localization in Zebrafish}

\author{
Rafael Trevisan*, Daniel Uzochukwu and Richard T. Di Giulio \\ Nicholas School of the Environment, Duke University, Durham, NC, United States
}

Plastics are world-wide pollutants that pose a potential threat to wildlife and human health. Small plastic particles, such as microplastics and nanoplastics, are easily ingested, and can act as a Trojan Horse by carrying microorganisms and pollutants. This study investigated the potential role of the Trojan Horse effect in the toxicity of nanoplastics to the vertebrate model organism, zebrafish (Danio rerio). First, we investigated if this effect could affect the toxicity of nanoplastics. Second, we analyzed if it could contribute to the biodistribution of the associated contaminants. And third, we focused on its effect on the mitochondrial toxicity of nanoplastics. We incubated $44 \mathrm{~nm}$ polystyrene nanoparticles with a real-world mixture of polycyclic aromatic hydrocarbons (PAHs) for 7 days and removed the free PAHs by ultrafiltration. We dosed embryos with 1 ppm of nanoplastics (NanoPS) or PAH-sorbed nanoplastics (PAH-NanoPS). Neither type of plastic particle caused changes in embryonic and larval development. Fluorescence microscopy and increased EROD activity suggested the uptake of PAHs in larvae exposed to PAH-NanoPS. This coincided with higher concentrations in the yolk sac and the brain. However, PAH-only exposure leads to their accumulation in the yolk sac but not in the brain, suggesting that that the spatial distribution of bioaccumulated PAHs can differ depending on their source of exposure. Both nanoplastic particles affected mitochondrial energy metabolism but caused different adverse effects. While NanoPS decreased NADH production, PAH-NanoPS decreased mitochondrial coupling efficiency and spare respiratory capacity. In summary, the addition of PAHs to the surface of nanoplastics did not translate into increased developmental toxicity. Low levels of PAHs were accumulated in the organisms, and the transfer of PAHs seems to happen in tissues and possibly organelles where nanoplastics accumulate. Disruption of the energy metabolism in the mitochondria may be a key factor in the toxicity of nanoplastics, and the Trojan Horse effect may amplify this effect.

Keywords: plastic pollution, nanoplastics, microplastics, carriers of organic pollutants, polystyrene, mitochondria, toxicity, zebrafish 


\section{INTRODUCTION}

Plastics are of significant concern for water contamination and constitute the largest amount of man-made debris in aquatic environments (Koelmans et al., 2015). Degradation of plastics via biotic and abiotic weathering leads to the formation of plastic particles at the micro $(\leq 5 \mathrm{~mm})$ and nanoscale $(\leq 1 \mu \mathrm{m})$ (Gigault et al., 2018). Concentrations of microplastics in freshwater and marine ecosystems are very variable, ranging from a few particles to thousands of particles per cubic meter (Hamid et al., 2018). Modeling studies suggest that microplastics ranging from 0.333 to $4.75 \mathrm{~mm}$ represent the vast majority of the plastic particles in the ocean, accounting for more than $90 \%$ of the total number of plastics in ocean surface waters (Eriksen et al., 2014). But modeling studies may underestimate the potential environmental threat of microplastics and nanoplastics. They are limited by the technical challenge on detecting small microplastics $(<0.333 \mathrm{~mm})$ and nanoplastics, the inadequate understanding of aggregation and deposition patterns for microplastics and nanoplastics, and the lack of robust models. For instance, a recent study (Brandon et al., 2019) indicated that microplastic levels can be 5-7 orders of magnitude higher than previously thought (Goldstein et al., 2013) when microplastics as small as $10 \mu \mathrm{m}$ are included. These values can reach a few million to tens of millions of particles per cubic meter in open ocean waters and nearshore areas (Brandon et al., 2019). These findings suggest that small microplastic particles, and presumably nanoplastics, are very abundant plastic particles and may account for a significant fraction of the problem of plastic pollution. Due to the small size of nanoplastics, detection and removal prove to be difficult, and it facilitates ingestion, tissue penetration, trophic transfer, and interactions with organic matter and other contaminants. Research addressing organismal accumulation and associated adverse effects is of crucial importance. Nanoplastics are probably the least understood compound of marine litter but potentially also the most hazardous one (Koelmans et al., 2015).

The toxicity of nanoplastics has been studied using different animal models, including the zebrafish (Danio rerio). Research indicates that polystyrene nanoparticles (NanoPS) smaller than $200 \mathrm{~nm}$ are able to cross the zebrafish embryonic chorion through pore canals and they appear to accumulate at first in the yolk sac, brain, retina, and blood vessels (Pitt et al., 2018a; Lee et al., 2019). NanoPS are then further transported to different organs, such as the heart, pancreas, gall bladder, liver, and the intestine. They can ultimately cause bradycardia and larval hypolocomotion (Pitt et al., 2018a). Uptake of nanoplastics can lead to disturbances in energy metabolism, including impaired glucose homeostasis and mitochondrial ATP production (Brun et al., 2019; Trevisan et al., 2019). These findings show that nanoplastics accumulate in early developmental stages, reach different organs throughout development, and have the potential to cause physiological disorders. Most of these results come from animals exposed to nanoplastics through the water. But nanoplastics can also be transferred through the diet (Chae et al., 2018) and via maternal transfer (Pitt et al., 2018b). Thus, their small size can also result in multiple routes of exposure.
Nanoplastics are generally composed of inert polymers but the adsorption of various molecules to their surfaces creates a coat called the surface corona. This has the potential to greatly increase their biological reactivity. The corona can consist of metal ions, polysaccharides, proteins, lipids, nucleic acids, microorganisms, and organic pollutants (PaulPont et al., 2018). Their high surface area and significant affinity to hydrophobic compounds suggest that nanoplastics will likely have organic compounds sorbed to their surface in the environment (Koelmans et al., 2015). Thus, nanoplastics can carry toxic environmental pollutants through a Trojan Horse effect mechanism. For example, plastic particles have a high affinity for hydrophobic organic contaminants such as polycyclic aromatic hydrocarbons (PAHs) (Lee et al., 2014). This Trojan Horse effect can be potentiated by specialized feeding strategies such as filter feeding, what can significantly increase the ingestion of plastic particles and associated contaminants (Fossi et al., 2014). In a previous study from our group, we found that NanoPS interact with a complex mixture of PAHs. This results in decreased levels of free PAHs available for uptake and reduces the acute toxicity of the NanoPS + PAHs mixture to zebrafish embryos (Trevisan et al., 2019). The present study aimed to investigate the sole contribution of the Trojan Horse effect to the transfer of PAHs. This was achieved by using an approach in which NanoPS are the only source of PAHs. We exposed zebrafish embryos to virgin or PAH-sorbed nanoplastics and analyzed their development, accumulation of PAHs, and mitochondrial energy metabolism. We hypothesized that plastics could expose the sorbed contaminants to aging and weathering, resulting in altered toxicity. We also hypothesized that exposure to PAH-sorbed plastics would cause signs similar to PAH exposure, such as cardiotoxicity, increased EROD activity, and PAH bioaccumulation. And as both nanoplastics and PAHs can modulate energy metabolism, we hypothesized nanoplastics loaded with PAHs could cause greater disruption of mitochondrial energy production when compared to virgin nanoplastics.

\section{MATERIALS AND METHODS}

\section{Plastic Nanoparticles and an Environmental Mixture of PAHs}

Non-functionalized polystyrene nanoparticles (NanoPS) were obtained from Bangs Laboratories, Inc. (Fishers, IN, United States), as a $10 \%$ (w:v) stock solution containing $0.1 \%$ sodium dodecyl sulfate (SDS) and $0.05 \%$ sodium azide. According to the manufacturer, the stock solution (100 ppt) contains $2.158 \mathrm{E}+15$ particles $/ \mathrm{ml}$. These same NanoPS were recently characterized in exposure medium (30\% Danieau) by our research group, with a hydrodynamic diameter of $44.73 \mathrm{~nm}$, a surface charge of $-38.0 \mathrm{mV}$, a relatively low polydispersity index (0.328), and polymer composition of polystyrene (Trevisan et al., 2019).

The environmental mixture of PAHs consisted of a sediment extract from the Atlantic Wood Industries Superfund site, located in the Elizabeth River (VA, United States), called Elizabeth River 
Sediment Extract (ERSE). It was previously collected, processed, and chemically characterized by our laboratory with a total PAH content of $5,073 \mathrm{ng} / \mathrm{mL}$ PAHs as a result of 36 different analyzed PAHs (Fang et al., 2014). This solution has been extensively studied in our laboratory as a model environmental PAH mixture with different fish species, including zebrafish (D. rerio), Atlantic killifish (Fundulus heteroclitus) and medaka (Oryzias latipes) (Brown et al., 2016; Riley et al., 2016; Lindberg et al., 2017; Mu et al., 2017; Trevisan et al., 2019). This extract was obtained from sediments collected at the Atlantic Wood Industries Superfund Site (VA, United States), which contained $\sim 122.6 \mu \mathrm{g} / \mathrm{g}$ dry sediment of total PAHs (Clark et al., 2013). These values are extremely high and close to the values obtained in highly industrialized or urbanized areas across the globe as reviewed by Clark and Di Giulio (2015): Tokyo Bay ( $300 \mu \mathrm{g} / \mathrm{g}$ dry weight), Sidney Harbor $(\sim 400 \mu \mathrm{g} / \mathrm{g}$ dry sediment), and Boston Harbor ( $\sim 400 \mu \mathrm{g} / \mathrm{g}$ dry sediment).

\section{Animal Husbandry and Embryo Collection}

Laboratory zebrafish (D. rerio) were maintained in a recirculating Aquatic Habitats system (Pentair Aquatic Eco-systems, Apopka, FL, United States) on a 14:10 h light: dark cycle. Water quality was maintained at $27.5-28.5^{\circ} \mathrm{C}$ and $\mathrm{pH} 7.0-7.5$, through the use of carbon-filtered water supplemented with commercial sea salts (60 mg/L; Instant Ocean, Foster \& Smith, Rhinelander, WI, United States). The fish were fed twice daily with brine shrimp (INVE Aquaculture, Inc., Salt Lake City, UT, United States) in the morning and Zeigler's Adult Zebrafish Complete Diet (Zeigler Bros., Inc., Gardners, PA, United States) in the afternoon. Breeding crosses of two males and three females were set at 5 $\mathrm{PM}$ and embryos were collected the following morning within $1 \mathrm{~h}$ of spawning between 9 and $10 \mathrm{AM}$ and kept in a medium of $30 \%$ Danieau water (17 mM NaCl, $2 \mathrm{mM} \mathrm{KCl,} 0.12 \mathrm{mM} \mathrm{MgSO}_{4}$, $1.8 \mathrm{mM} \mathrm{Ca}\left(\mathrm{NO}_{3}\right)_{2}, 1.5 \mathrm{mM}$ HEPES, $\mathrm{pH}$ 7.6).

\section{Sorption of PAHs to the Surface of NanoPS}

For each exposure independent experiment, two glass flasks containing $40 \mathrm{ml}$ of $30 \%$ Danieau water were prepared 1 week before the experiment and NanoPS were added at the final concentration of $10 \mathrm{ppm}$. One of the flasks also received the ERSE solution at the final concentration of 5\% (PAH-NanoPS group), while the other flask did not receive ERSE (NanoPS group). These values were chosen based on data from a previous experiment that suggested the sorption of PAHs to NanoPS at these concentrations (Trevisan et al., 2019). The flasks were incubated at $28^{\circ} \mathrm{C}$ and $60 \mathrm{rpm}$ for 7 days with a $14: 10 \mathrm{~h}$ light: dark cycle (Figure 1A), after which the solutions were transferred to Vivaspin 300 KDa MWC filters (Figure 1B) and centrifuged as recommended by the manufacturer. The samples were washed three times with 30\% Danieau to remove free PAHs and the retained fraction containing the nanoplastics was resuspended in 30\% Danieau to the nominal concentration of $1 \mathrm{ppm}$ NanoPS and 1 ppm PAH-NanoPS. At this concentration, the expected number of particles is $2.158 \mathrm{E}+9$ particles $/ \mathrm{ml}$, close to the highest predicted environmental concentrations for $50 \mathrm{~nm}$ nanoplastics particles (between $1 \mathrm{E}+3$ to $1 \mathrm{E}+9$ particles/L) (Lenz et al., 2016).

\section{Exposure to NanoPS and PAH-NanoPS}

Zebrafish embryos at $6 \mathrm{hpf}$ (hours post-fertilization) were dosed with freshly prepared NanoPS or PAH-NanoPS solutions as described in section "Sorption of PAHs to the Surface of NanoPS," and an additional group was kept in the exposure solution (30\% Danieau) as a control (Ctl) (Figures 1C,D). The exposures were carried out in glass Petri dishes at a density of 1 zebrafish embryo/ml. The total number of embryos per group varied according to each assay, as described in the following sections. Animals were exposed to the solutions in an incubator at $28^{\circ} \mathrm{C}$ and $60 \mathrm{rpm}$ until further analysis.

\section{Exposure to Different Ultrafiltration Fractions of the Fresh and Aged PAH Mixture}

Preliminary experiments were carried out to account for possible interferences in the toxicity assays. To investigate if the 7-day pre-incubation period (hereafter called aging) could affect the toxicity of the PAH-NanoPS solution due to alterations in the chemical structure of the PAHs, preliminary acute toxicity assays were carried out with unfiltered (total fraction) 5\% fresh ERSE and 5\% aged ERSE (Figures 1E,F). The aged ERSE solution was prepared in 30\% Danieau under the same conditions as described in section "Sorption of PAHs to the Surface of NanoPS": one flask containing $40 \mathrm{ml}$ of 5\% ERSE (diluted in 30\% Danieau water) was prepared 1 week before the experiment. The flask was incubated at $28^{\circ} \mathrm{C}$ and $60 \mathrm{rpm}$ for 7 days with a $14: 10 \mathrm{~h}$ light: dark cycle. Animals were then exposed to this solution (unfiltered 5\% aged ERSE) or freshly prepared unfiltered 5\% ERSE (freshly prepared in $30 \%$ Danieau).

Another set of experiments was performed to investigate the possible accumulation of free PAHs in the filter, which could contaminate and increase the toxicity of the PAH-NanoPS solution. Acute toxicity assays were tested with the retained and eluted fractions of 5\% fresh and aged ERSE (Figures 1E,F): $30 \mathrm{ml}$ of freshly prepared 5\% ERSE (diluted in 30\% Danieau) or 5\% aged ERSE (prepared as described above) were transferred to Vivaspin $300 \mathrm{KDa}$ MWC filters, centrifuged as recommended by the manufacturer, and the eluted fraction was collected. The retained fraction was washed three times with 30\% Danieau to remove free PAHs and resuspended in $30 \mathrm{ml}$ of 30\% Danieau. Animals were exposed to these solutions as described above.

\section{Preliminary Assessment of the Developmental Toxicity of Fresh and Aged ERSE Solutions}

Zebrafish embryos exposed to the total, retained, and eluted fractions of freshly prepared or aged ERSE solutions were analyzed for survival and deformity rates at 96 hpf. Each experiment consisted of 2 Petri dishes of 10 embryos per group, and each petri dish was considered a biological replicate in a total of three independent experiments $(n=6)$. Results 


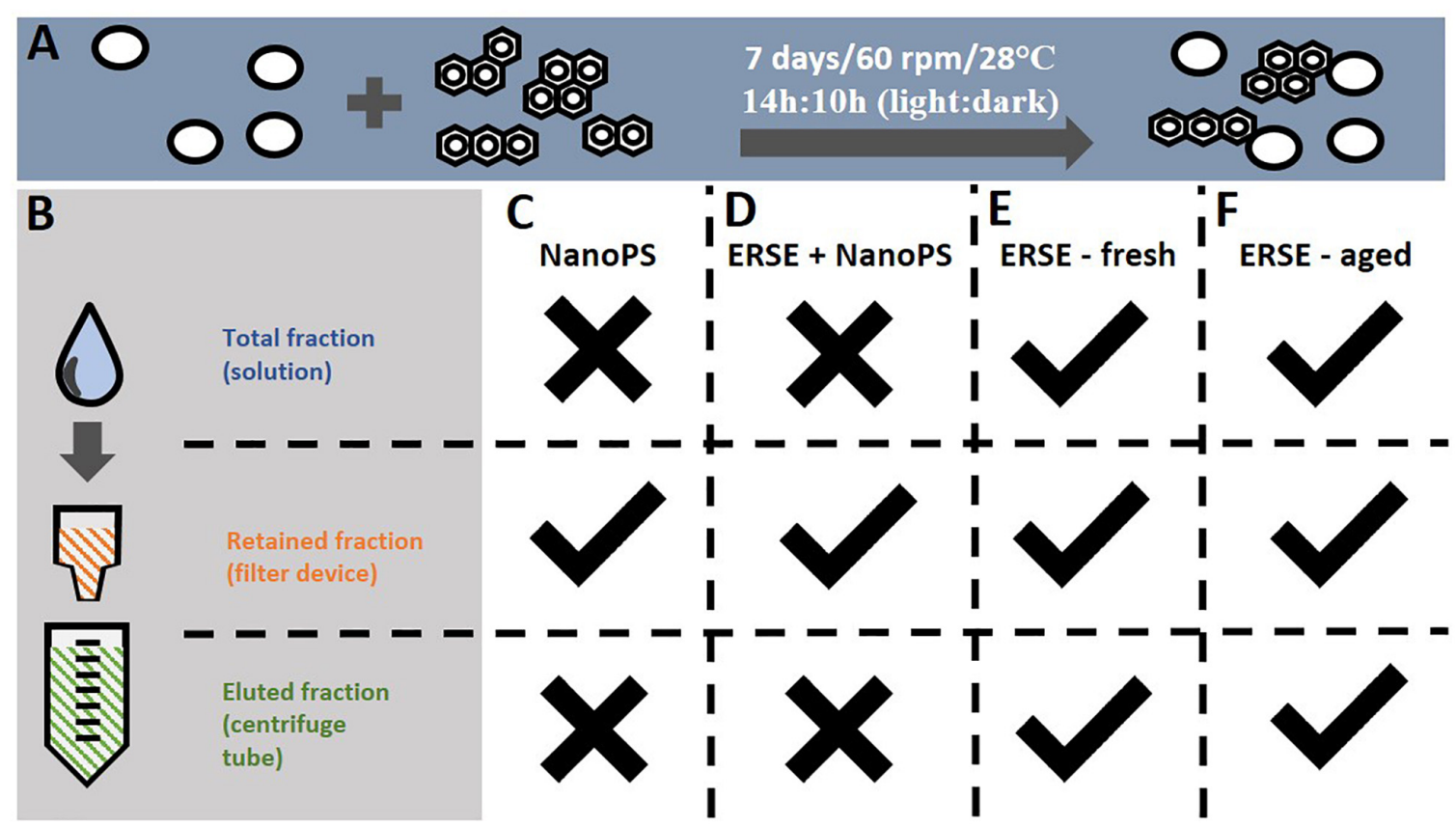

FIGURE 1 | Procedures for the sorption of PAHs to nanoplastics and the exposure groups analyzed in this study. (A) Polystyrene nanoparticles (44 nm) were incubated at $10 \mathrm{ppm}$ with a mixture of PAHs (Elizabeth River Sediment Extract, ERSE - 5\% v:v) in a glass flask for 7 days at the described conditions. Incubations containing only NanoPS or ERSE were carried out in the same conditions. (B) Free PAHs unbound to nanoplastics were removed from the solution using an ultrafiltration device, followed by resuspension of the retained nanoplastics to the final concentration of 1 ppm (NanoPS and PAH-NanoPS groups). (C,D) Zebrafish embryos were exposed only to the retained fractions of the NanoPS and PAH-NanoPS solutions. For comparison, embryos were also exposed to the total (unfiltered), retained and eluted fractions of (E) fresh 5\% ERSE (non-incubated) or (F) aged 5\% ERSE (incubated for 7 days in the absence of nanoplastics). Checkmark indicates that the respective fraction was analyzed for toxicity while an $\mathrm{X}$ indicates the opposite.

of these preliminary experiments were used for design of subsequent studies.

\section{Assessment of the Developmental Toxicity of NanoPS and PAH-NanoPS}

Zebrafish embryos exposed to 1 ppm NanoPS or PAH-NanoPS underwent a deformity assessment in the form of yolk sac edema, pericardial edema, and curved tail every $24 \mathrm{~h}$ until $96 \mathrm{hpf}$. The survival rate and hatching rate was determined at the same time points, except that the hatching rate was additionally analyzed at $56 \mathrm{hpf}$. Each experiment was carried out with three Petri dishes per group, and each petri dish was considered a biological replicate, in a total of three independent experiments $(n=9)$. The heartbeat rate was measured in these same experiments at $48 \mathrm{hpf}$ in 12 embryos per group (four embryos from each petri dish) $(n=36)$.

\section{Analysis of PAH Exposure by the EROD Assay}

In vivo Ethoxyresorufin-O-Deethylase (EROD) activity was analyzed in $96 \mathrm{hpf}$ zebrafish larvae to estimate $\mathrm{PAH}$ exposure in Ctl, NanoPS, and PAH-NanoPS exposure groups. At $24 \mathrm{hpf}$, embryos were dosed with $0.02 \mathrm{mg} / \mathrm{L}$ PTU (phenylthiourea) to inhibit pigmentation. At $96 \mathrm{hpf}$, embryos were individually transferred to solid white 384 well plates and incubated with
$1.5 \mu \mathrm{M}$ 7-ethoxyresorufin. The plate was incubated in a plate reader at $28^{\circ} \mathrm{C}$, and the fluorescence was quantified after $8 \mathrm{~h}$. The assay was adapted from a previously published method (Noury et al., 2006), and the relative EROD activity was calculated by normalizing the fluorescence values to the control group. The experiment was performed with 10 larvae per group per experiment, in three independent experiments $(n=30)$.

\section{Analysis of PAH Uptake by Fluorescence Microscopy}

$\mathrm{PAH}$ uptake was estimated in $96 \mathrm{hpf}$ zebrafish larvae in Ctl, NanoPS, and PAH-NanoPS exposure groups via fluoresce microscopy, utilizing the natural fluorescence of PAHs. At 24 hpf, embryos were dosed with $0.02 \mathrm{mg} / \mathrm{L}$ PTU (phenylthiourea) to inhibit pigmentation. Larvae were individually transferred to a black clear bottom 96 well plate, anesthetized in $200 \mu \mathrm{g} / \mathrm{mL}$ tricaine (prepared in 30\% Danieau), and analyzed for green fluorescence using a BZ-X700 automated fluorescence microscope (Keyence Corporation of America, Itasca, IL, United States) with 470/40 (em) and 525/50 nm (ex) filters. In vivo quantitative determination of $\mathrm{PAH}$ uptake was performed relative to the control group. Fluorescence was quantified using ImageJ software and expressed as fold change normalized to the control group. The experiment was performed with 6-7 larvae per group per experiment, in three independent experiments $(n=18-21)$. 
To estimate the concentration of PAHs bioaccumulated by the end of the PAH-NanoPS exposure, the fluorescence fold change obtained as described above was compared to the values obtained from animals exposed to unfiltered (total fraction) aged ERSE solution at the concentrations of $0.1,0.5,1,2$, and $5 \%$. This experiment was performed with four larvae per group per experiment, in three independent experiments $(n=12)$.

\section{Bioenergetics Analyses}

Assessment of oxygen consumption rate and extracellular acidification rate was performed via the XFe24 Extracellular Flux Analyzer (Agilent Instruments, Santa Clara, CA, United States) in $24 \mathrm{hpf}$ embryos or $96 \mathrm{hpf}$ larvae according to previously published protocols (Stackley et al., 2011; Raftery et al., 2017). Animals were staged in an islet capture microplate (24 wells) containing filtered water supplemented with commercial sea salts (65 ppm), at a density of two embryos or one larva per well. Carbonyl cyanide-4-(trifluoromethoxy)phenylhydrazone (FCCP), oligomycin A, and sodium azide were used to estimate the bioenergetics partitioning, as described in Supplementary Material. Each experiment consisted of 7-8 animals per group, in three independent experiments $(n=21-24)$.

Animals were also assayed in vivo for $\mathrm{NADH}$ production according to a previously published protocol using Alamar Blue (Thermo Fisher Scientific, Waltham, MA, United States) (Williams and Renquist, 2016). Embryos at $24 \mathrm{hpf}$ or larvae at $96 \mathrm{hpf}$ were transferred to a 96 well white plate at a density of two embryos per well or one larva per well, and the fluorescence was analyzed over $24 \mathrm{~h}$ at $28^{\circ} \mathrm{C}$. The change in fluorescence values between the end and the start of the assay was calculated, normalized to the control group for each experiment, and expressed as relative fluorescence change. Each experiment consisted of 10 animals per group, in three independent experiments $(n=21-24)$.

\section{Statistical Analysis}

Data were analyzed for normal distribution and homogeneity of variability by Shapiro-Wilk and Bartletts, respectively. Survival rate and deformity rates after exposure to different ultrafiltration fractions of fresh or aged ERSE or different concentrations of unfiltered aged ERSE were analyzed by Kruskal-Wallis followed by the two-stage set-up method of Benjamin, Krieger, and Yekutieli for controlling the false discovery rate (5\%). Additional experiments with NanoPS and PAH-NanoPS exposure groups were analyzed by one-way ANOVA followed by Tukey's posthoc or Kruskal-Wallis followed by Dunn's post-hoc. Values were considered statistically significant at $p<0.05$.

\section{RESULTS}

\section{Preliminary Assessment of Aging and Ultrafiltration in the Developmental Toxicity of the PAH Mixture}

Initial experiments investigated if the incubation period of 7 days at $28^{\circ} \mathrm{C}$ could cause aging and weathering effects on the PAHs, affecting their developmental toxicity. Zebrafish embryos were exposed to the total fraction (unfiltered) of freshly prepared or aged 5\% ERSE solution. The results shown in Figure $\mathbf{2}$ indicate that the total fraction of the fresh $\mathrm{PAH}$ solution is highly toxic to early development zebrafish. Exposure to it caused high frequencies of animals containing pericardial edemas (98\%) or curved tails (34\%). On the other hand, the toxicity of the aged PAHs solution appears to be lower. After exposure, fewer animals presented pericardial edema $(36 \%)$, but a similar number of them had curved tails (28\%).

The effects of the removal of free PAHs by ultrafiltration on the toxicity of the fresh and aged ERSE solution were analyzed. This experiment aimed to investigate if PAHs could unintentionally accumulate in the retained fraction during the ultrafiltration of the PAH-NanoPS solution. Such accumulation could contaminate and overestimate the biological effects of the PAH-NanoPS. No signs of toxicity were detected when animals were exposed to the retained fractions of fresh or aged PAHs solutions (survival rate $>86 \%$ and deformity rates $<3 \%$ ). These findings suggest that no significant amount of PAHs accumulates in this fraction after the washing steps. Yet, the toxicity of the eluate fraction was slightly lower than the total fraction for both PAHs solutions, probably due to some loss of free PAHs during the ultrafiltration.

\section{Developmental Toxicity Assays With NanoPS and PAH-NanoPS}

The preliminary tests ruled out the significant accumulation of free PAHs in the retained fraction. After such confirmation, an initial daily screening for alterations in developmental and morphological parameters was performed in zebrafish embryos and larvae exposed to 1 ppm NanoPS or PAH-NanoPS (Figure 3). Results indicated no significant differences for survival, hatching, and deformity rates from 24 to $96 \mathrm{hpf}$. No signs of cardiotoxicity were detected when the heartbeat was assayed in $48 \mathrm{hpf}$ animals (Figure 3).

We measured the larval EROD activity as a qualitative indication of the transfer and bioaccumulation of PAHs. A 1.8fold increase in EROD activity was detected in larvae exposed to PAH-NanoPS (Figure 3). Despite the absence of classic signs of PAH developmental toxicity, this result suggests that bioaccumulation of PAHs occurs after exposure to PAH-NanoPS.

\section{PAH Uptake in Organisms Exposed to Nano-PS or PAH-NanoPS}

We used the natural fluorescence of PAHs to further investigate the bioaccumulation of PAHs. Green fluorescence levels were analyzed in $96 \mathrm{hpf}$ larvae through fluorescence microscopy. In line with the EROD data, we detected high fluorescence levels in animals exposed to PAH-NanoPS (Figures 4A,B). A 3D surface plot analysis of the green fluorescence (Figure 4C) indicates the yolk sac as the main organ for PAH bioaccumulation. This same analysis also indicates that the area corresponding to the brain appears as a possible target. Quantitative analysis indicates a 2.6fold increase in the fluorescence levels in the whole animal, a 


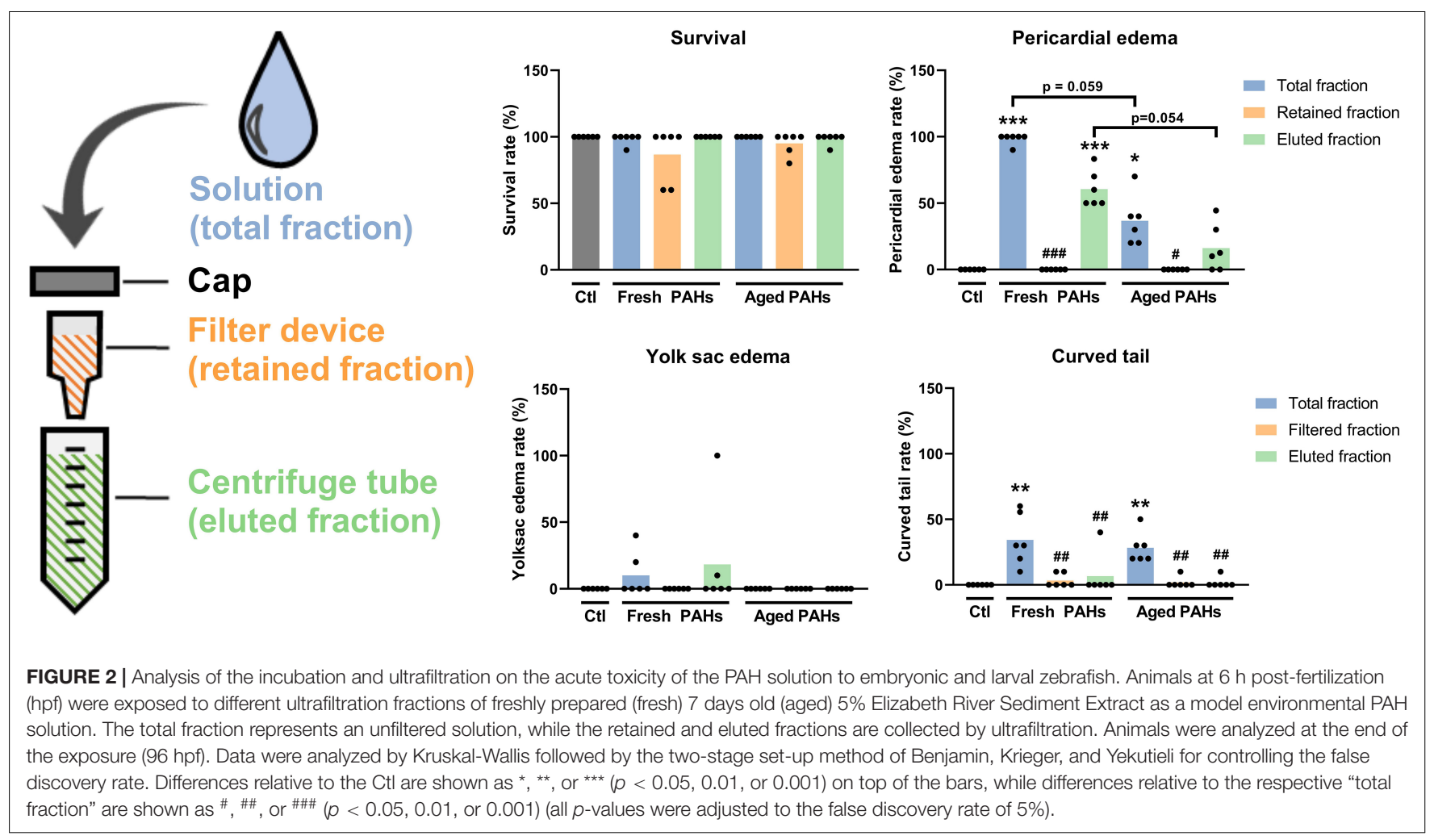

4.0-fold increase in the yolk sac, and a 1.25 -fold increase in the brain region of larvae exposed to PAH-NanoPS.

We also estimated the amount of PAHs accumulated in larvae exposed to PAH-NanoPS (Figure 4D). The fluorescence levels of organisms exposed to PAH-NanoPS were compared to the ones obtained from direct exposures to aged ERSE. The fluorescence increase in the whole animal and the yolk sac in PAH-NanoPS animals is similar to the increase after exposure to $0.5 \%$ aged ERSE. Yet, we did not detect significant accumulation of PAHs in the brain region with aged ERSE concentration as high as 5\%. Moreover, the levels of total PAHs accumulated after exposure to 5\% aged ERSE were still slightly lower than the ones from PAH-NanoPS animals.

\section{Mitochondrial Bioenergetics of Animals Exposed to NanoPS and PAH-NanoPS}

Both nanoplastics and PAHs can affect the mitochondria (Meyer et al., 2013; Lee et al., 2019; Trevisan et al., 2019). Thus, we analyzed the mitochondrial energy metabolism in zebrafish embryos and larvae in vivo. A summary of the mitochondrial bioenergetics partitioning can be seen in Figure 5. The top panel (Figure 5A) shows the different metabolic fractions of the oxygen consumption rates (OCR) analyzed in vivo in this study. The total maximum respiration of the organism is comprised of a non-mitochondrial fraction, a mitochondrial basal respiration fraction, and a mitochondrial spare capacity fraction. We can also divide the mitochondrial basal respiration into ATP-linked and proton leak fractions. The bioenergetics fractions in $24 \mathrm{hpf}$ embryos and $96 \mathrm{hpf}$ larvae are shown as the corresponding percentage of the total maximum respiration (sum equals 100\%). The statistical analyses of the absolute values of these fractions are shown in Supplementary Figures S1, S2.

Figure 5B indicates the effects of NanoPS and PAH-NanoPS on the bioenergetics fractions in $24 \mathrm{hpf}$ embryos. Both exposures seem to increase the fraction associated with proton-leak at the expense of ATP-linked OCR. This can be further analyzed by looking at the mitochondrial coupling efficiency. This parameter represents the percentage of mitochondrial respiration linked to ATP synthesis. Only exposure to PAH-NanoPS caused a significant decrease, from 85 to $79 \%$ (Figure 5C).

It is not possible to estimate the proton-leak and ATP-linked OCR in $96 \mathrm{hpf}$ larvae. Under our experimental conditions, oligomycin A is not effective at this developmental stage. Thus, only the basal mitochondrial OCR fraction is shown. At this developmental stage, PAH-NanoPS decreased the mitochondrial spare capacity from 29 to $21 \%$ of the total maximum respiration. It also decreased the non-mitochondrial OCR fraction from 12.7 to $9.2 \%$ (Figure 5D).

In addition to the OCRs, we measured the NADH production and extracellular acidification rate (ECAR) in vivo in embryos and larvae. In these whole organisms, the ECAR values do not reflect the glycolysis rate due to lactic acid excretion. They are indicative of the excretion of $\mathrm{CO}_{2}$ from the tricarboxylic acid (TCA) cycle (Stackley et al., 2011). $\mathrm{CO}_{2}$ will then generate bicarbonate and carbonate in the medium, changing the $\mathrm{pH}$ value of the medium. We did not see differences in either of these assays in $24 \mathrm{hpf}$ embryos (Figure 6). Yet, $96 \mathrm{hpf}$ larvae had lower NADH production rates when exposed to NanoPS. It was not possible 


\section{Survival rate}

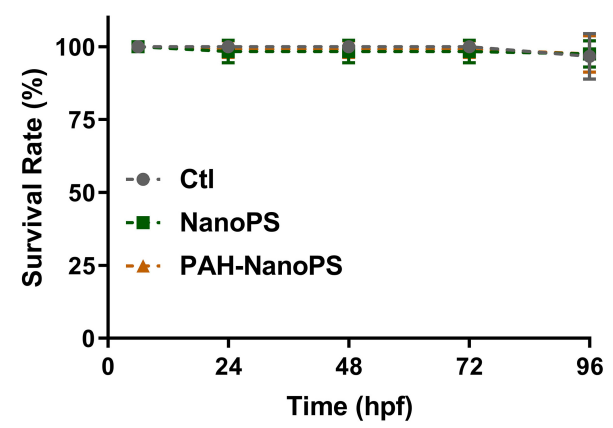

Pericardial edema rate

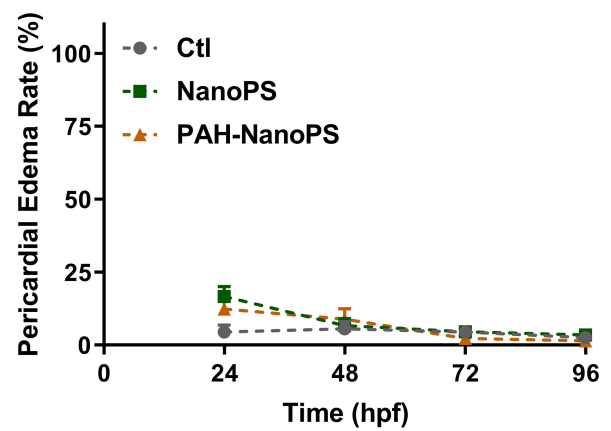

Heartbeat - $48 \mathrm{hpf}$

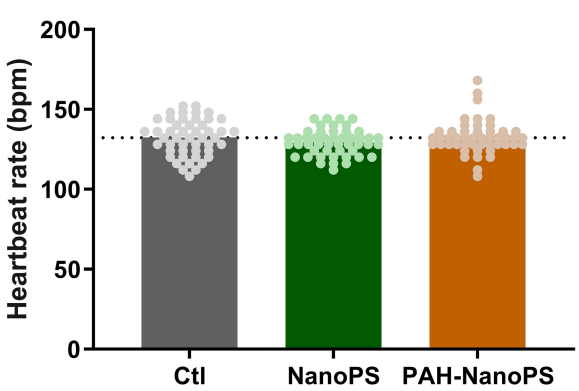

Hatching rate

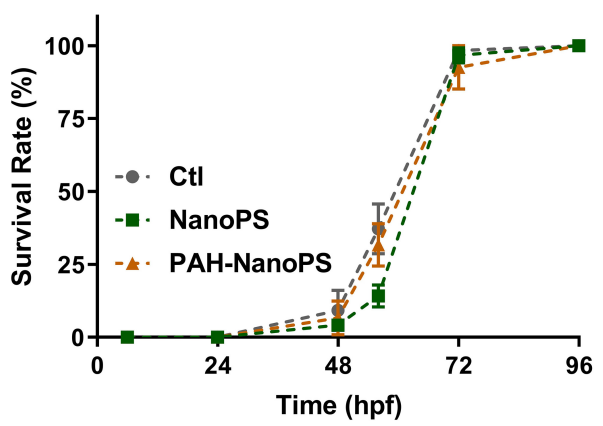

Yolk sac edema rate

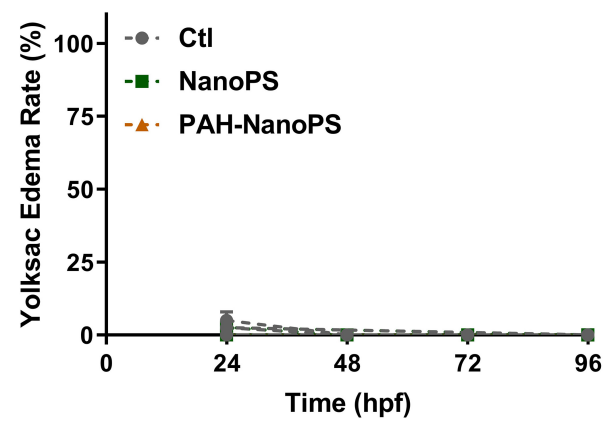

EROD activity - 96 hpf

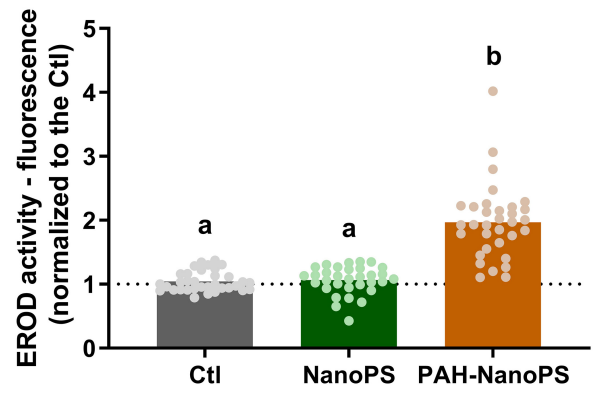

FIGURE 3 | Morphological screening and EROD activity of zebrafish embryos and larvae. Animals were exposed at 6 h post-fertilization (hpf) to 1 ppm polystyrene nanoparticles (NanoPS) or 1 ppm polystyrene nanoparticles with PAHs sorbed to their surface (PAH-NanoPS) (please see Figure 1 and section "Sorption of PAHs to the surface of NanoPS" for further details). Survival, hatching, yolk sac edema, and pericardial edema were assessed throughout the exposure, while the heartbeat was assessed at $48 \mathrm{hpf}$ and EROD activity was analyzed in vivo at $96 \mathrm{hpf}$. Data are presented as average \pm standard deviation ( $n=9-12)$, except for heartbeat $(n=48)$ and EROD activity $(n=32-35)$ which are shown as average and scatter plot of individual replicates. The dotted line represents the average of the control group. Survival rate was analyzed by Mantel-Cox log-rank test; hatching, yolk sac edema, and pericardial edema rates were individually analyzed at each time point by one-way ANOVA followed by Tukey's post-hoc or Kruskal-Wallis followed by Dunn's post-hoc; heartbeat was analyzed by one-way ANOVA followed by Tukey's post-hoc. Groups not sharing letters are statistically different $(p<0.01)$.

to measure the ECAR/CO 2 release rates in larvae, as the values were negligible.

\section{DISCUSSION}

As previously discussed, plastics have been detected globally in a wide range of environments, including freshwater and marine environments. Land-based activities are major players for marine plastic pollution. Thus, it is not surprising that rivers are classified as main transporters of plastics into the estuaries and the ocean, and that estuarine benthic sediments are important sinks for plastic accumulation (Simon-Sánchez et al., 2019). During this transport, the sorption of organic pollutants to plastic particles can result in the accumulation of chemicals in the estuaries (Fraser et al., 2020) and ultimately, the ocean. In freshwater systems, nanoplastics quickly interact with dissolved organic matter to form microgels, which will be transformed 
A

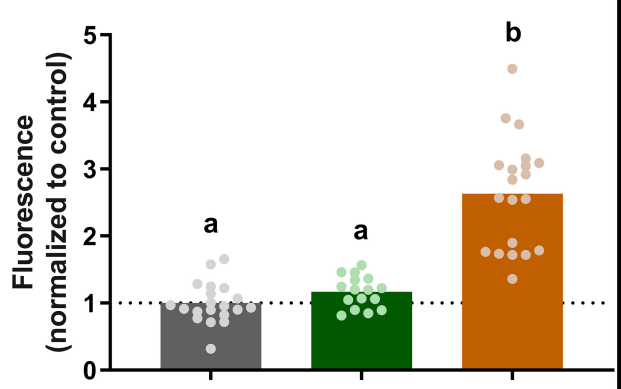

Yolk Sac

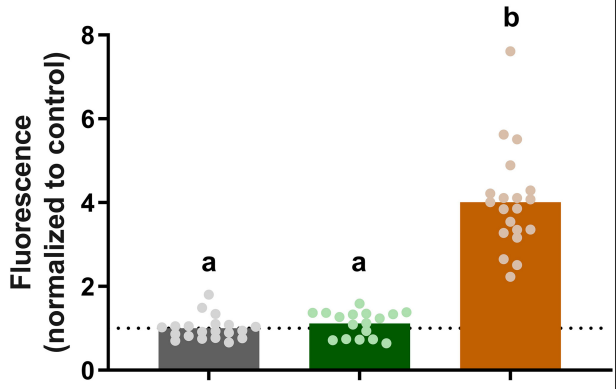

Brain region

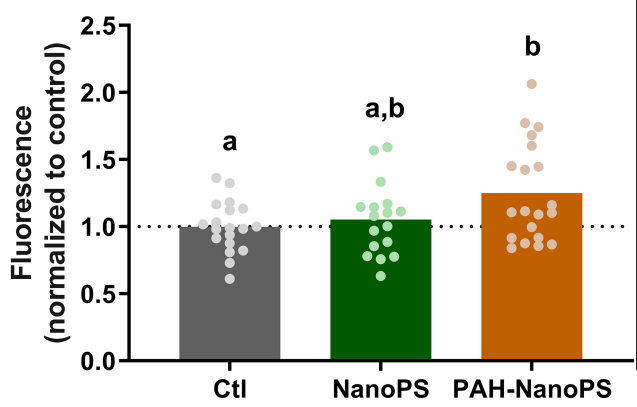

D

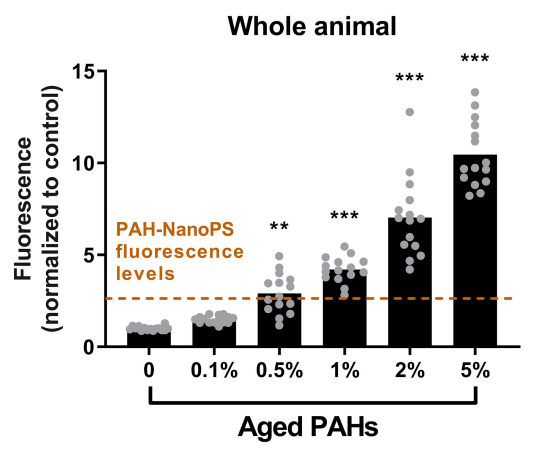

B Representative images

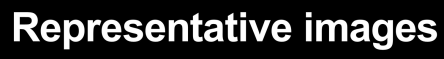

C- Ctl

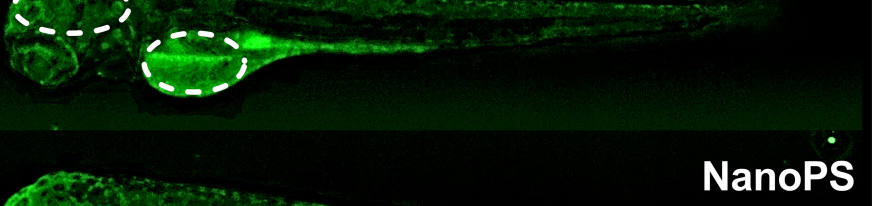

(1) $)^{\circ}=$

C

Detailed PAH localization

PAH-NanoPS

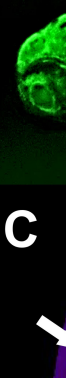

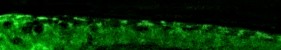

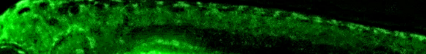

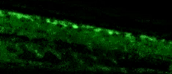
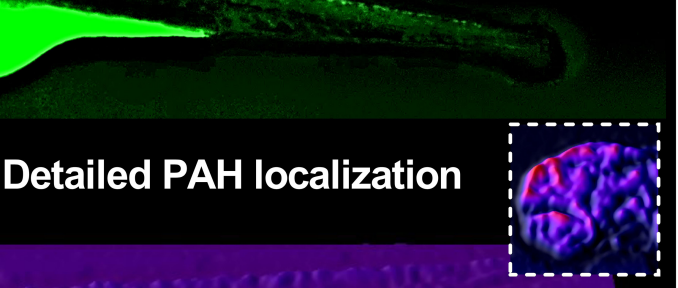

CtI

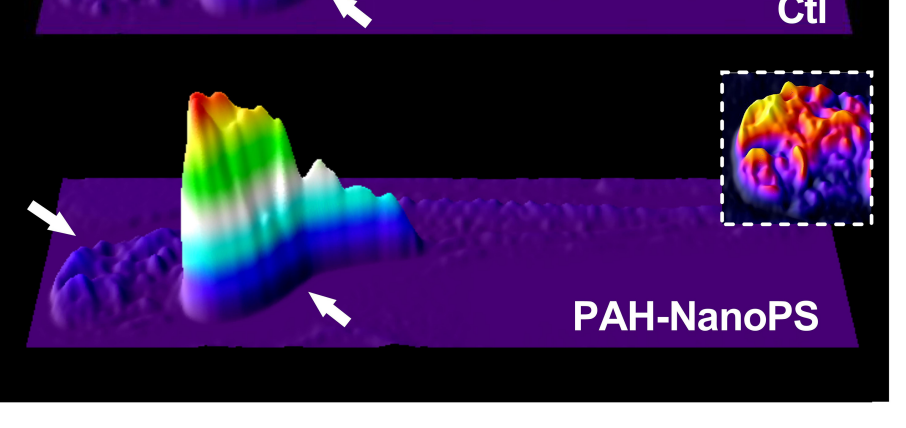

$$
\text { (2) }
$$
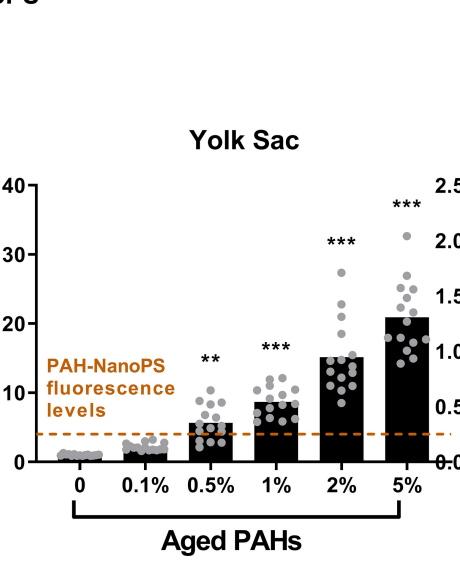

Yolk Sac

Brain region

FIGURE 4 | PAH uptake estimated by green fluorescence microscopy in 96 hpf larvae. Animals were exposed at 6 h post-fertilization (hpf) to 1 ppm polystyrene nanoparticles (NanoPS) or 1 ppm polystyrene nanoparticles with PAHs sorbed to their surface (PAH-NanoPS) (see Figure 1 and section "Sorption of PAHs to the surface of NanoPS" for further details). (A) Green fluorescence was quantified in the whole animal, in the yolk sac, and in the brain region, and is shown as average and scatter plot of individual replicates $(n=17-20)$. (B) Representative images are shown in the top, and the dashed circles indicate the individual areas analyzed. (C) 3D surface plot representations of the green fluorescence levels indicate that the fluorescence increase related to PAHs uptake occurs mostly in the yolk sac (arrow) and to some extent in the brain region (arrow). The insets (dashed line) represent an estimate of PAH uptake in the brain region with the color scale adjusted (Continued) 
FIGURE 4 | Continued

to that region. (D) Green fluorescence levels in larvae exposed to aged ERSE $(n=15)$. The black dotted line represents the average of the control group, while the dashed orange lines indicate the fluorescence values obtained from animals exposed to PAH-NanoPS. Data were analyzed by one-way ANOVA followed by Tukey's post-hoc or Kruskal-Wallis followed by Dunn's post-hoc for (A), and one-way ANOVA followed by Dunnet's post-hoc or Kruskal-Wallis followed by Dunn's post-hoc for (D). Groups not sharing letters are statistically different $(p<0.01)$ when compared to the Ctl group (A), and ${ }^{* \star}(p<0.01)$ or *** $(p<0.001)$ represents statistical differences against the Ctl group (D).

A

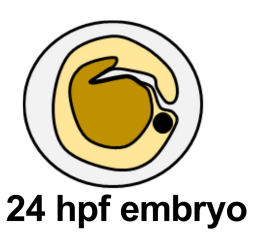

C

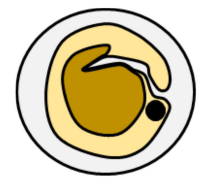

24 hpf embryo

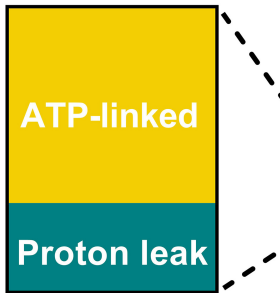

\section{Mitochondrial spare capacity}

Mitochondrial

basal respiration

Non-mitochondrial

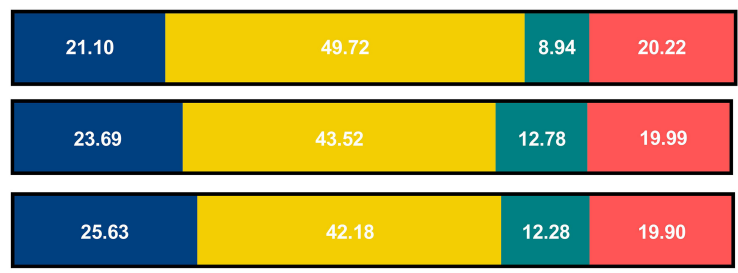

Mitochondrial coupling efficiency

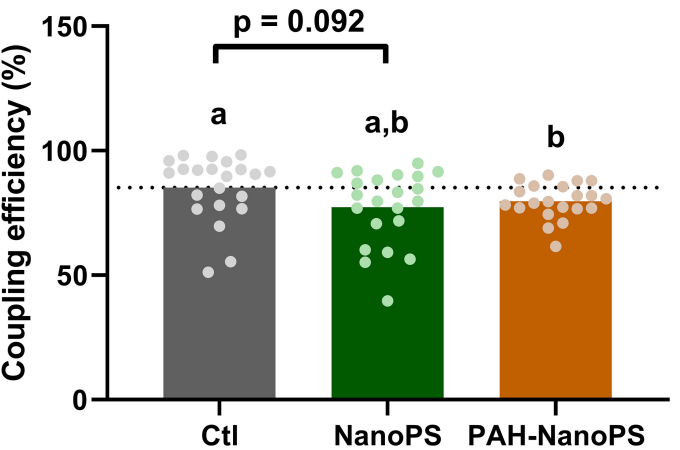

D

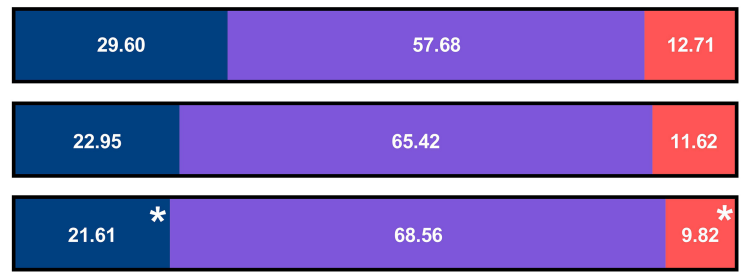

CtI

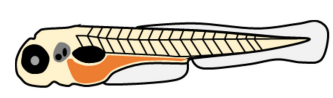

96 hpf larva
21.61
08.50
CtI

NanoPS

PAH-NanoPS

Total maximum respiration

FIGURE 5 | Mitochondrial bioenergetics profiles of zebrafish embryos and larvae. Animals were exposed at $6 \mathrm{~h}$ post-fertilization (hpf) to 1 ppm polystyrene nanoparticles (NanoPS) or 1 ppm polystyrene nanoparticles with PAHs sorbed to their surface (PAH-NanoPS) (please see Figure 1 and section "Sorption of PAHs to the surface of NanoPS" for further details). (A) In vivo oxygen consumption rates (OCR) in 24 hpf embryos, shown as the proportion of OCR due to the mitochondrial spare capacity, ATP synthase (ATP-linked), proton leak, and non-mitochondrial respiration. The percentages were calculated based on the in vivo total maximal OCR values (100\%, sum of non-mitochondrial, mitochondrial basal and spare capacity). (B) Mitochondrial coupling efficiency of oxidative phosphorylation in 24 hpf zebrafish embryos, shown as average and the scatter plot of individual replicates $(n=21-22)$. The dotted line represents the average of the control group. (C) $I n$ vivo oxygen consumption rates (OCR) in 96 hpf larvae, shown as the proportion of OCR due to mitochondrial spare capacity, basal mitochondrial respiration (sum of ATP-linked and proton leak), and non-mitochondrial respiration. ATP-linked respiration, proton leak respiration, and mitochondrial coupling efficiency could not be determined in larvae due to their lack of response to the chemical drugs used in the assay. (D) Metabolic partitioning of whole animal OCR used in the present study. For the bioenergetics partitioning, ${ }^{*}$ represents statistical difference when compared to the control group $(p<0.05)$, while for the mitochondrial coupling efficiency groups not sharing letters indicate statistical differences $(p<0.05)$. Please see Supplementary Material for additional information on the assay, equations for the bioenergetics partitioning, and additional statistical analyses. 
In vivo NADH production - 24 hpf

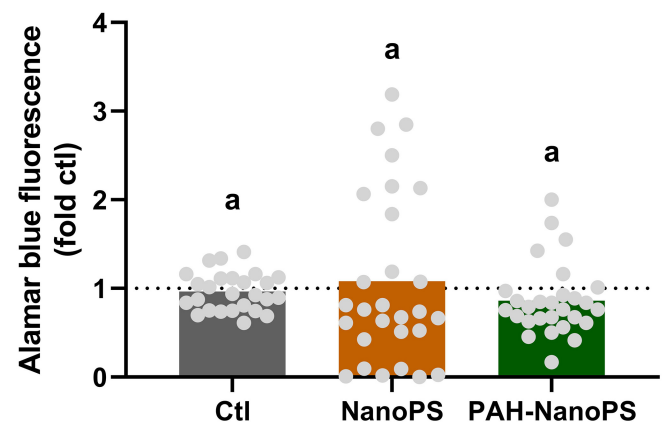

In vivo $\mathrm{CO}_{2}$ excretion - $24 \mathrm{hpf}$

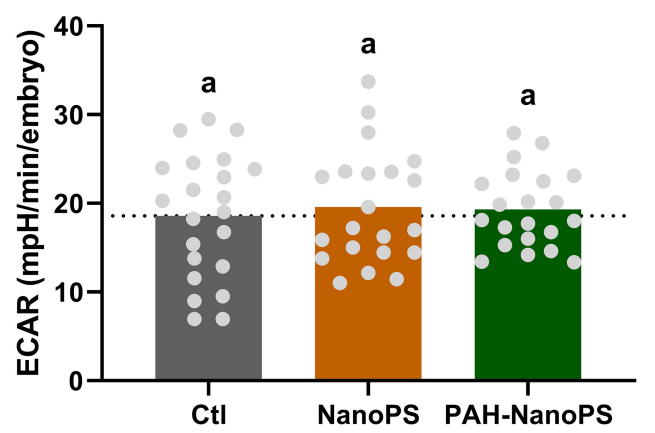

In vivo NADH production - 96 hpf
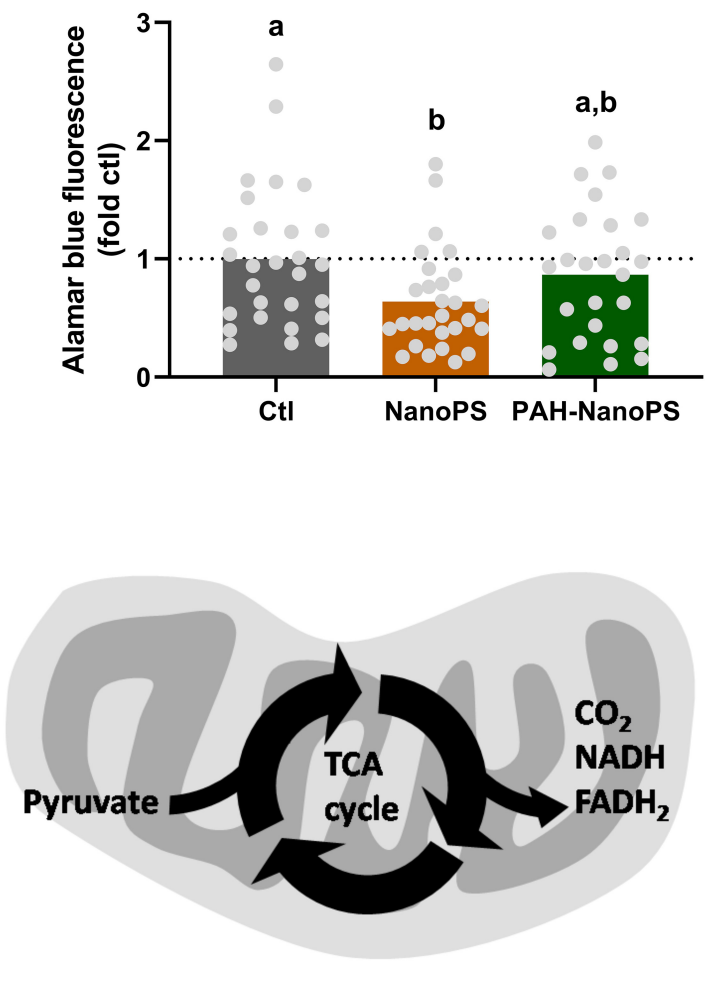

FIGURE 6 | Assessment of the TCA cycle through NADH production and $\mathrm{CO}_{2}$ release in zebrafish embryos and larvae. Animals were exposed at $6 \mathrm{~h}$ post-fertilization (hpf) to 1 ppm polystyrene nanoparticles (NanoPS) or 1 ppm polystyrene nanoparticles with PAHs sorbed to their surface (PAH-NanoPS) (please see Figure 1 and section "Sorption of PAHs to the surface of NanoPS" for further details). In vivo NADPH production was estimated in 24 hpf embryos and 96 hpf larvae ( $n=26-28$ ), while $\mathrm{CO}_{2}$ release from the TCA cycle was analyzed only in 24 hpf embryos $(n=21-22)$. The dotted line represents the average of the control group. The bottom right image indicates how NADH and CO2 productions can be indicative of the activity of the TCA cycle. Data were analyzed by one-way ANOVA followed by Tukey's post-hoc or Kruskal-Wallis followed by Dunn's post-hoc. Groups not sharing letters are significantly different ( $p<0.05)$.

into particulate organic matter and facilitate sedimentation and pollutant transport (Shiu et al., 2020). Despite this role of rivers in the fate and transport of microplastics and co-contaminants, there is a large gap between research on freshwater and marine plastic pollution as little is known about plastic particles in freshwater systems. This study aims to provide information on how nanoplastics can be a source of other pollutants in freshwater systems and potentially in estuaries and marine environments. It aims to describe how the transport of such contaminants by nanoplastics in these environments can affect the energy metabolism of embryonic and larval fish, as we used zebrafish as a model organism commonly used to investigate the potential adverse effects of freshwater and marine contaminants.

Many studies have shown that virgin nanoplastics can cause adverse effects in a range of organisms. This includes bacteria, algae, zooplankton, bivalves, copepods, and fish, as reviewed elsewhere (Shen et al., 2019). Many authors suggest that the use of virgin and homogeneous micro or nanoplastics is a limitation for toxicological studies (Horton et al., 2017). But such studies can provide valuable information for future research using other plastic particles and more realistic exposure scenarios. For example, many studies using virgin plastics have investigated the potential role of the Trojan Horse effect. And they have shown that it can increase the overall toxicity of plastics particles (Ma et al., 2016; Yu et al., 2019). Previous data from our group indicated that NanoPS decreases the uptake and the overall toxicity of free PAHs in a co-exposure scenario. This is likely due to the sorption of the PAHs to the NanoPS (Trevisan et al., 2019). The present study further explored this topic by investigating if exposures to PAH-sorbed NanoPS could cause the transfer of PAHS and induce signs of PAHdependent toxicity in developing zebrafish. In specific, we were interested if such transfer would happen in areas suggested to accumulate NanoPS during early development. And we aimed to investigate if such bioaccumulation could lead to disruption of the energy metabolism.

Our experimental design used nanoplastics as the only source of PAHs during the exposure. The exposure of nanoplastics to PAHs happened at 5\% ERSE, $\sim 253.65 \mu \mathrm{g} / \mathrm{L}$. These values are considered high when compared to the PAHs levels detected in rivers in urban or industrial areas across the world, such as the Seine River (France, 0.004-0.036 $\mu \mathrm{g} / \mathrm{L}$ ), Mississippi River (United States, 0.063-0.145 $\mu \mathrm{g} / \mathrm{L}$ ), Tonghui River (China, 0.192-2.651 $\mu \mathrm{g} / \mathrm{L}$ ), and Pearl River (China, 6.960-26.920 $\mu \mathrm{g} / \mathrm{L}$ ) 
(reviewed by Cao et al., 2010), but can be found in highly contaminated estuarine and marine environments such as the Niger Delta (Nigeria, 24.390-283.600 $\mu \mathrm{g} / \mathrm{L}$ ) (Duke, 2008). It is important to note that the total levels of PAHs detected in each study are a result of the PAHs selected for analysis, and therefore the values cannot be easily compared between studies. In our experimental approach, after the sorption of PAHs to the nanoplastics, we removed any remaining free PAHs from solution by ultrafiltration. Preliminary toxicity assays estimated the efficiency of such removal. The results indicated no signs of acute toxicity in the retained fraction of both the fresh or aged ERSE solution in the absence of nanoplastics. This finding suggests that the levels of free PAHs were low or absent after the washing steps. Thus, the use of ultrafiltration should not interfere with the toxicity of the PAH-NanoPS particles. In fact, we detected a possible loss of free PAHs after the ultrafiltration. This is expected as PAHs can sorb to the filter resin (polyethersulfone) (Endo and Matsuura, 2018). But it is important to highlight that this should not affect the toxicity of the PAH-NanoPS solution, as it should not contain free PAHs after the ultrafiltration.

The effects of the 7 days incubation period on the toxicity of the PAHs solution were also analyzed. The aged ERSE solution was less toxic to developing zebrafish than the respective fresh solution. Aging and weathering effects such as volatilization, dissolution, and photodegradation can have direct effects on PAHs. They can decrease their concentrations, change their physicochemical properties, and alter the final composition of PAH mixtures (Stout et al., 2016). The exact mechanisms underlying the lower toxicity of the aged ERSE solution were not investigated in the present study. But our results suggest that weathering and aging of the associated pollutants must be taken into account for laboratory studies. They also point to a significant role of these events on the toxicity of plastics in the environment.

The initial toxicity screening of NanoPS and PAH-NanoPS indicated no signs of adverse effects of these particles. We did not detect changes in the survival, hatching, and deformity rates in zebrafish using the same NanoPS in previous studies (Pitt et al., 2018a; Trevisan et al., 2019). A decrease in heartbeat rate after exposure to $1 \mathrm{ppm}$ NanoPS has been reported previously by our group (Pitt et al., 2018a). These results cannot be easily compared to the present study, in which the NanoPS were pre-incubated for 7 days. But these findings suggest that $35-45 \mathrm{~nm}$ NanoPS at the low ppm range do not cause major effects on zebrafish early development. Other studies of NanoPS of similar sizes had comparable results (Lee et al., 2019; Liu et al., 2019).

Sorption of NanoPS with PAHs for 7 days before the exposure period did not lead to acute toxicity or teratogenicity. The environmental mixture of PAHs used in this study (ERSE) is a potent inducer of cardiac toxicity in developing fish (Brown et al., 2016; Riley et al., 2016; Lindberg et al., 2017; Mu et al., 2017; Trevisan et al., 2019). The lack of such findings in animals exposed to PAH-NanoPS suggests, at least, two possible scenarios. One is the low transfer of PAHs through exposure to PAHNanoPS. And the other is the decrease of the PAH-related toxicity of the PAH-NanoPS due to aging and weathering during the incubation period.
Analysis of EROD activity indicated a possible increase in the body burden of PAHs in animals exposed to PAH-NanoPS. ERSE exposure is known to cause an increase in EROD activity through the activation of the AhR pathway (Fleming and Di Giulio, 2011; Lindberg et al., 2017; Trevisan et al., 2019). Although it does not necessarily correspond to the developmental toxicity of PAHs, EROD activity is a very sensitive biomarker response to exposure to many PAHs (Shankar et al., 2019). Fluorescence microscopy confirmed the bioaccumulation of PAHs in animals exposed to PAH-NanoPS. It occurred mostly in the yolk sac and at minor levels in the brain. This increase in PAH levels in the whole animal and the yolk sac is equivalent to exposures between 0.1 and $0.5 \%$ of aged ERSE. At these concentrations, aged ERSE is not expected to affect the development of zebrafish embryos (Supplementary Figure S3). This supports the acute toxicity data obtained from the PAH-NanoPS group. Fluorescence microscopy cannot discriminate between PAHs accumulated as free molecules or still sorbed to the nanoplastics. It remains unclear what is the extent of PAH desorption after the uptake of PAH-NanoPS. Regardless, EROD activity serves as a biomarker for increased bioaccumulation of reactive PAHs. Our findings show that, at least, a significant fraction of the bioaccumulated PAHs should be available as free PAHs within the zebrafish. It also remains unclear which PAHs present on the ERSE mixture sorb to the nanoplastics and bioaccumulated in the organisms. And how aging and weathering may have affected the PAH composition of the plastic particles and the profile of PAHs bioaccumulated. Further studies on this topic could offer insights into the organic contaminant buildup in aquatic environments and biota by nano and microplastics.

The bioaccumulation of PAHs in the yolk sac and the brain is not surprising. Both organs can be a target for hydrophobic contaminants due to their high lipid content. An interesting finding was that no significant amount of PAHs bioaccumulated in the brain of larvae exposed to aged ERSE at concentrations as high as $5 \%$. This is the opposite of the data obtained from animals exposed to PAH-NanoPS. PAHs seem to bioaccumulate at higher levels in the brain when the exposure happens through nanoplastics rather than through the water. Nanoplastics accumulate at high levels in the yolk sac and can be further transported to other organs, including the brain and digestive organs (Pitt et al., 2018a; Sokmen et al., 2019). This should ease the transfer of associated contaminants to these organs. Unfortunately, the bioaccumulation of PAHs in the digestive organs of larval zebrafish was not investigated due to the high fluorescence levels in the yolk sac in animals exposed to PAHNanoPS. Co-exposures of nano and microplastics with other chemicals can alter the biodistribution of the co-contaminants (Chen et al., 2017; Trevisan et al., 2019). Ma et al. (2016) showed nanoplastics accumulate in the gut of daphnids and that coexposures with phenanthrene increase the accumulation of this PAH in the whole animal (Ma et al., 2016). However, these studies relied on co-exposures of plastics and other contaminants. They could not address if the Trojan Horse effect of plastic particles could cause the transfer of contaminants to specific areas of the organisms. This possibility of targeted transfer of associated contaminants needs to be further investigated. Such an effect can 
help to understand the potential risks of plastic particles in a more realistic environmental scenario.

Mitochondria are also targets of both nanoparticles and PAHs (Meyer et al., 2013; Jayasundara, 2017), and our results show that both NanoPS and PAH-NanoPS cause mitochondrial stress. The effects of NanoPS were limited to decreased NADH production in larvae. On the other hand, PAH-NanoPS decreased embryonic mitochondrial coupling efficiency and larval mitochondrial spare capacity. Virgin NanoPS at higher concentration (10 ppm) can decrease embryonic mitochondrial coupling efficiency and increase larval NADH production in zebrafish (Trevisan et al., 2019). Nanoplastics smaller than $100 \mathrm{~nm}$ can be easily taken up by cells. After uptake, they interact with the tubulin cytoskeleton and localize within the mitochondria (Johnston et al., 2010). Live-cell imaging indicates that exposure to virgin NanoPS causes rapid uptake and accumulation of nanoplastics in the cytosol. This is followed by interaction and accumulation in reticular and vesicular structures such as mitochondria. Diffusion to the nucleus can also happen, but at a much slower rate (Hemmerich and Von Mikecz, 2013). The mitochondrial toxicity of polystyrene nanoparticles is not limited to effects on energy metabolism. It also includes mitochondrial swelling, loss of mitochondrial membrane integrity, and release of cytochrome c. This toxicity depends on nanoparticle's surface chemistry and corona (Ruenraroengsak and Tetley, 2015).

The effects on mitochondrial bioenergetic fractions happened only in animals exposed to PAH-NanoPS. PAHs can decrease ATP production and content by negatively affecting mitochondrial coupling (Du et al., 2015; Lindberg and Di Giulio, 2019). PAH exposure can also decrease mitochondrial spare capacity in vitro and in vivo (Bansal et al., 2014; Raftery et al., 2017). As the effects on these parameters were limited to the exposure to $\mathrm{PAH}$-sorbed polystyrene nanoparticles, we suggest that the Trojan Horse effect can also determine the fate of associated contaminants at the subcellular level. It is possible that nanoplastics can transfer environmental pollutants to target organelles, including the mitochondria. The release of these chemicals could then cause more severe adverse effects. Mitochondria are associated with numerous metabolic pathways, not only with ATP production and the TCA cycle, but also fatty acid oxidation, amino acid metabolism, nitrogen balance and elimination, calcium signaling, and the biosynthesis of heme, steroid hormones, pyrimidines and purines ( $\mathrm{Hu}$ et al., 2019). The potential toxicity of nanoplastics and associated contaminants to the mitochondria suggest that exposure to these plastic particles can have direct effects on many cellular systems. For example, exposure to virgin NanoPS decreased the levels of metabolites related to the TCA cycle and amino acid metabolism in the nematode Caenorhabditis elegans (Kim et al., 2019). It also disrupted the expression of genes associated with energy metabolism in the copepod Daphnia pulex (Liu et al., 2018). But further studies are necessary to shed light on the effects of nanoplastics on mitochondrial metabolism and their physiological consequences, and how the Trojan Horse effect can aggravate this scenario.

As recently suggested, there is an urgent need for a more comprehensive view of ecotoxicology with a focus on the biological fitness of an organism instead of lethal and sublethal effects (Straub et al., 2020). While additional studies with multigenerational and reproductive approaches would be necessary to achieve such a goal, this study indicates that energy production can be a key component of the ecological impacts of pollutant-sorbed nanoplastics. It is known that mitochondrial integrity and function are essential for organismal performance and fitness and that mitochondria can be more sensitive to pollutants than natural stressors (Sokolova, 2018). Impaired mitochondrial bioenergetics can lead to decreased larval growth (Bolser et al., 2018), altered larval behavior (Zhang et al., 2017), and possibly lower reproduction rates (as discussed by Dreier et al., 2019). These are key ecological outcomes linked to animal fitness. Based on our data and the use of mitochondrial Adverse Outcome Pathways (as reviewed by Dreier et al., 2019), we hypothesize that the exposure of freshwater organisms to high concentrations of pollutant-sorbed nanoplastics (at the ppm range) can potentially impact the ecosystem via the disruption of energy homeostasis at the organismal level. And that long-term exposures to this scenario could cause populational effects by decreased reproduction rates and population decline. It is important to note that in estuarine and marine environments, sedimentation of nanoplastics due to salinity and particulate organic matter can partially protect free-swimming organisms from waterborne exposures to nanoplastics. But it remains unclear if the trophic transfer of nanoplastics and associated contaminants or their accumulation in sediments in these environments could trigger similar effects in energy homeostasis of free-swimming or sessile organisms, respectively.

In summary, our results suggest that aging and weathering events can play an important role in plastic toxicity. They can not only affect the plastic particles but also the chemical structure and composition of contaminants bound to them. This can result in different toxicity profiles and should be taken into consideration for laboratory and field studies. The sorption of PAHs to nanoplastics has significant effects on their toxicity. In this study, it did not affect fish early development, but as expected, nanoplastics acted as carriers of environmental pollutants. Like nanoplastics, PAHs accumulated in lipid-rich environments. It occurred primarily and in the yolk sac, but significant levels were also found in the brain. We hypothesize that the Trojan Horse effect can also happen at the tissue and organelle level, with plastic particles mediating the transfer of PAHs to specific parts of the organism. The sorption of PAHs also increased the toxicity of the nanoplastics to the mitochondria through a decrease in their efficiency to produce energy. Our findings also indicate that developing fish exposed to nanoplastics maintain their ability to survive. However, the presence of PAHs in their surface could potentially affect the nervous system and the ability of organisms to operate in high-energy demand scenarios.

\section{DATA AVAILABILITY STATEMENT}

The datasets generated for this study are available on request to the corresponding author. 


\section{ETHICS STATEMENT}

The animal study was reviewed and approved by the Duke University Animal Care and Use Program.

\section{AUTHOR CONTRIBUTIONS}

RT designed the experiment and wrote the main manuscript. RT and DU performed the experiments. RD contributed with the reagents, materials, and analysis tools. All authors contributed to the article and approved the submitted version.

\section{FUNDING}

The research was supported by the Duke's Superfund Research Center (NIEHS P42-ES010356), the National Science Foundation (NSF), and the Environmental Protection Agency (EPA)

\section{REFERENCES}

Bansal, S., Leu, A. N., Gonzalez, F. J., Guengerich, F. P., Chowdhury, A. R., Anandatheerthavarada, H. K., et al. (2014). Mitochondrial targeting of cytochrome P450 (CYP) 1B1 and its role in polycyclic aromatic hydrocarboninduced mitochondrial dysfunction. J. Biol. Chem. 289, 9936-9951. doi: 10. 1074/jbc.m113.525659

Bolser, D. G., Dreier, D. A., Li, E., Kroll, K. J., Martyniuk, C. J., and Denslow, N. D. (2018). Toward an adverse outcome pathway for impaired growth: mitochondrial dysfunction impairs growth in early life stages of the fathead minnow (Pimephales promelas). Comp. Biochem. Physiol. CToxico. Pharmacol. 209, 46-53. doi: 10.1016/j.cbpc.2018. 03.009

Brandon, J. A., Freibott, A., and Sala, L. M. (2019). Patterns of suspended and salp-ingested microplastic debris in the North Pacific investigated with epifluorescence microscopy. Limnol. Oceanogr. Lett. 5, 46-53. doi: 10.1002/lol2. 10127

Brown, D. R., Bailey, J. M., Oliveri, A. N., Levin, E. D., and Di Giulio, R. T. (2016). Developmental exposure to a complex PAH mixture causes persistent behavioral effects in naive Fundulus heteroclitus (killifish) but not in a population of PAH-adapted killifish. Neurotoxicol. Teratol. 53, 55-63. doi: 10.1016/j.ntt.2015.10.007

Brun, N. R., Van Hage, P., Hunting, E. R., Haramis, A. G., Vink, S. C., Vijver, M. G., et al. (2019). Polystyrene nanoplastics disrupt glucose metabolism and cortisol levels with a possible link to behavioural changes in larval zebrafish. Commun. Biol. 2:382.

Cao, Z., Liu, J., Li, Y., and Ma, M. (2010). Distribution and source apportionment of polycyclic aromatic hydrocarbons (PAH) in water and sediments of the Luan River. China. Toxicol. Environ. Chem. 92, 717-720.

Chae, Y., Kim, D., Kim, S. W., and An, Y. J. (2018). Trophic transfer and individual impact of nano-sized polystyrene in a four-species freshwater food chain. Sci. Rep. 8:284.

Chen, Q. Q., Yin, D. Q., Jia, Y. L., Schiwy, S., Legradi, J., Yang, S. Y., et al. (2017). Enhanced uptake of BPA in the presence of nanoplastics can lead to neurotoxic effects in adult zebrafish. Sci. Total Environ. 609, 1312-1321. doi: 10.1016/j. scitotenv.2017.07.144

Clark, B. W., Cooper, E. M., Stapleton, H. M., and Di Giulio, R. T. (2013). Compound- and mixture-specific differences in resistance to PAHs and PCB126 among Fundulus heteroclitus subpopulations throughout the Elizabeth River estuary (Virginia. USA). Environ. Sci. Technol. 47, 10556-10566. under NSF Cooperative Agreement EF-0830093 and DBI1266252, Center for the Environmental Implications of Nanotechnology (CEINT).

\section{ACKNOWLEDGMENTS}

We thank all members of the Di Giulio laboratory for help with zebrafish husbandry. We are thankful for Omar Saleh for helping with the toxicity screening assays. We also thank Drs. Danielle Ferraz Mello and Lindsay Jasperse for their help with the manuscript revision.

\section{SUPPLEMENTAL MATERIAL}

The Supplementary Material for this article can be found online at: https://www.frontiersin.org/articles/10.3389/fenvs. 2020.00078/full\#supplementary-material

Clark, B. W., and Di Giulio, R. T. (2015). The elizabeth river story: a case study in evolutionary toxicology. J. Toxicol. Environ. Health B Cri. Rev. 18, 259-298. doi: 10.1080/15320383.2015.1074841

Dreier, D. A., Mello, D. F., Meyer, J. N., and Martyniuk, C. J. (2019). Linking mitochondrial dysfunction to organismal and population health in the context of environmental pollutants: progress and considerations for mitochondrial adverse outcome pathways. Environ. Toxicol. Chem. 38, 1625-1634.

Du, X., Crawford, D. L., and Oleksiak, M. F. (2015). Effects of anthropogenic pollution on the oxidative phosphorylation pathway of hepatocytes from natural populations of Fundulus heteroclitus. Aquat. Toxicol. 165, 231-240. doi: 10.1016/j.aquatox.2015.06.009

Duke, O. (2008). Source determination of polynuclear aromatic hydrocarbons in water and sediment of a creek in the Niger Delta region. Afr. J. Biotechnol. 7, $282-285$.

Endo, S., and Matsuura, Y. (2018). Characterizing sorption and permeation properties of membrane filters used for aquatic integrative passive samplers. Environ. Sci. Technol. 52, 2118-2125. doi: 10.1021/acs.est.7b0 5144

Eriksen, M., Lebreton, L. C. M., Carson, H. S., Thiel, M., Moore, C. J., Borerro, J. C., et al. (2014). Plastic pollution in the world's oceans: more than 5 trillion plastic pieces weighing over 250,000 tons afloat at sea. PLoS One 9:e111913. doi: 10.1371/journal.pone.0111913

Fang, M., Getzinger, G. J., Cooper, E. M., Clark, B. W., Garner, L. V., Di Giulio, R. T., et al. (2014). Effect-directed analysis of Elizabeth River porewater: developmental toxicity in zebrafish (Danio rerio). Environ. Toxicol. Chem. 33, 2767-2774. doi: 10.1002/etc. 2738

Fleming, C. R., and Di Giulio, R. T. (2011). The role of CYP1A inhibition in the embryotoxic interactions between hypoxia and polycyclic aromatic hydrocarbons (PAHs) and PAH mixtures in zebrafish (Danio rerio). Ecotoxicology 20, 1300-1314. doi: 10.1007/s10646-011-0686-1

Fossi, M. C., Coppola, D., Baini, M., Gianetti, M., Guerranti, C., Marsili, L., et al. (2014). Large filter feeding marine organisms as indicators of microplastic in the pelagic environment: the case studies of the Mediterranean basking shark (Cetorhinus maximus) and fin whale (Balaenoptera physalus). Mar. Environ. Res. 100, 17-24. doi: 10.1016/j.marenvres.2014. 02.002

Fraser, M. A., Chen, L., Ashar, M., Huang, W., Zeng, J., Zhang, C., et al. (2020). Occurrence and distribution of microplastics and polychlorinated biphenyls in sediments from the Qiantang River and Hangzhou Bay. China. Ecotoxicol. Environ. Saf. 196:110536. doi: 10.1016/j.ecoenv.2020.110536 
Gigault, J., Halle, A. T., Baudrimont, M., Pascal, P. Y., Gauffre, F., Phi, T. L., et al. (2018). Current opinion: what is a nanoplastic? Environ. Pollut. 235, 1030-1034. doi: 10.1016/j.envpol.2018.01.024

Goldstein, M. C., Titmus, A. J., and Ford, M. (2013). Scales of spatial heterogeneity of plastic marine debris in the northeast Pacific ocean. PLoS One 8:e80020. doi: 10.1371/journal.pone.0080020

Hamid, F. S., Bhatti, M. S., Anuar, N., Anuar, N., Mohan, P., and Periathamby, A. (2018). Worldwide distribution and abundance of microplastic: how dire is the situation? Waste Manag. Res. 36, 873-897. doi: 10.1177/0734242x1878 5730

Hemmerich, P. H., and Von Mikecz, A. H. (2013). Defining the subcellular interface of nanoparticles by live-cell imaging. PLoS One 8:e62018. doi: 10.1371/ journal.pone.0062018

Horton, A. A., Walton, A., Spurgeon, D. J., Lahive, E., and Svendsen, C. (2017). Microplastics in freshwater and terrestrial environments: evaluating the current understanding to identify the knowledge gaps and future research priorities. Sci. Total Environ. 586, 127-141. doi: 10.1016/j.scitotenv.2017. 01.190

Hu, X., Go, Y. M., and Jones, D. P. (2019). Omics integration for mitochondria systems biology. Antioxid Redox Signal 32, 853-872. doi: 10.1089/ars.2019.8006 Jayasundara, N. (2017). Ecological significance of mitochondrial toxicants. Toxicology 391, 64-74. doi: 10.1016/j.tox.2017.07.015

Johnston, H. J., Semmler-Behnke, M., Brown, D. M., Kreyling, W., Tran, L., and Stone, V. (2010). Evaluating the uptake and intracellular fate of polystyrene nanoparticles by primary and hepatocyte cell lines in vitro. Toxicol. Appl. Pharmacol. 242, 66-78. doi: 10.1016/j.taap.2009. 09.015

Kim, H. M., Lee, D. K., Long, N. P., Kwon, S. W., and Park, J. H. (2019). Uptake of nanopolystyrene particles induces distinct metabolic profiles and toxic effects in Caenorhabditis elegans. Environ. Pollut. 246, 578-586. doi: 10.1016/j.envpol. 2018.12.043

Koelmans, A. A., Besseling, E., and Shim, W. J. (2015). Nanoplastics in the aquatic environment. Crit. Rev. Mar. Anthropogen. Litter 2018, 325-340. doi: 10.1007/ 978-3-319-16510-3_12

Lee, H., Shim, W. J., and Kwon, J. H. (2014). Sorption capacity of plastic debris for hydrophobic organic chemicals. Sci. Total Environ. 470, 1545-1552. doi: 10.1016/j.scitotenv.2013.08.023

Lee, W. S., Cho, H. J., Kim, E., Huh, Y. H., Kim, H. J., Kim, B., et al. (2019). Bioaccumulation of polystyrene nanoplastics and their effect on the toxicity of $\mathrm{Au}$ ions in zebrafish embryos. Nanoscale 11, 3396-3396. doi: 10.1039/ c8nr90280a

Lenz, R., Enders, K., and Nielsen, T. G. (2016). Microplastic exposure studies should be environmentally realistic. Proc. Natl. Acad. Scie. U.S.A. 113, E4121E4122.

Lindberg, C. D., and Di Giulio, R. T. (2019). Polycyclic aromatic hydrocarbon and hypoxia exposures result in mitochondrial dysfunction in zebrafish. Aquat. Toxicol. 216:105298. doi: 10.1016/j.aquatox.2019.105298

Lindberg, C. D., Jayasundara, N., Kozal, J. S., Leuthner, T. C., and Di Giulio, R. T. (2017). Resistance to polycyclic aromatic hydrocarbon toxicity and associated bioenergetic consequences in a population of Fundulus heteroclitus. Ecotoxicology 26, 435-448. doi: 10.1007/s10646-0171775-6

Liu, Y. H., Wang, Z., Wang, S., Fang, H., Ye, N., and Wang, D. G. (2019). Ecotoxicological effects on Scenedesmus obliquus and Danio rerio Coexposed to polystyrene nano-plastic particles and natural acidic organic polymer. Environ. Toxicol. Pharmacol. 67, 21-28. doi: 10.1016/j.etap.2019. 01.007

Liu, Z. Q., Cai, M. Q., Yu, P., Chen, M. H., Wu, D. L., Zhang, M., et al. (2018). Age-dependent survival, stress defense, and AMPK in Daphnia pulex after short-term exposure to a polystyrene nanoplastic. Aquat. Toxicol. 204, 1-8. doi: 10.1016/j.aquatox.2018.08.017

Ma, Y. N., Huang, A. N., Cao, S. Q., Sun, F. F., Wang, L. H., Guo, H. Y., et al. (2016). Effects of nanoplastics and microplastics on toxicity, bioaccumulation, and environmental fate of phenanthrene in fresh water. Environ. Pollut. 219, 166-173. doi: 10.1016/j.envpol.2016.10.061

Meyer, J. N., Leung, M. C. K., Rooney, J. P., Sendoel, A., Hengartner, M. O., Kisby, G. E., et al. (2013). Mitochondria as a target of environmental toxicants. Toxicol. Sci. 134, 1-17. doi: 10.1093/toxsci/kft102
Mu, J. L., Chernick, M., Dong, W., Di Giulio, R. T., and Hinton, D. E. (2017). Early life co-exposures to a real-world PAH mixture and hypoxia result in later life and next generation consequences in medaka (Oryzias latipes). Aquat. Toxicol. 190, 162-173. doi: 10.1016/j.aquatox.2017. 06.026

Noury, P., Geffard, O., Tutundjian, R., and Garric, J. (2006). Non destructive in vivo measurement of ethoxyresorufin biotransformation by zebrafish prolarva: development and application. Environ. Toxicol. 21, 324-331. doi: 10.1002/tox. 20184

Paul-Pont, I., Tallec, K., Gonzalez-Fernandez, C., Lambert, C., Vincent, D., Mazurais, D., et al. (2018). Constraints and priorities for conducting experimental exposures of marine organisms to microplastics. Front. Mar. Sci. 5:252. doi: 10.3389/fmars.2018.00252

Pitt, J. A., Kozal, J. S., Jayasundara, N., Massarsky, A., Trevisan, R., Geitner, N., et al. (2018a). Uptake, tissue distribution, and toxicity of polystyrene nanoparticles in developing zebrafish (Danio rerio). Aquat. Toxicol. 194, 185-194. doi: 10.1016/ j.aquatox.2017.11.017

Pitt, J. A., Trevisan, R., Massarsky, A., Kozal, J. S., Levin, E. D., and Di Giulio, R. T. (2018b). Maternal transfer of nanoplastics to offspring in zebrafish (Danio rerio): a case study with nanopolystyrene. Sci. Total Environ. 643, 324-334. doi: 10.1016/j.scitotenv.2018.06.186

Raftery, T. D., Jayasundara, N., and Di Giulio, R. T. (2017). A bioenergetics assay for studying the effects of environmental stressors on mitochondrial function in vivo in zebrafish larvae. Comp. Biochem. Physiol. C Toxicol. Pharmacol. 192, 23-32. doi: 10.1016/j.cbpc.2016.12.001

Riley, A. K., Chernick, M., Brown, D. R., Hinton, D. E., and Di Giulio, R. T. (2016). Hepatic responses of juvenile Fundulus heteroclitus from pollution-adapted and nonadapted populations exposed to Elizabeth River sediment extract. Toxicol. Pathol. 44, 738-748. doi: 10.1177/0192623316636717

Ruenraroengsak, P., and Tetley, T. D. (2015). Differential bioreactivity of neutral, cationic and anionic polystyrene nanoparticles with cells from the human alveolar compartment: robust response of alveolar type 1 epithelial cells. Part. Fibre Toxicol. 12:19.

Shankar, P., Geier, M. C., Truong, L., Mcclure, R. S., Pande, P., Waters, K. M., et al. (2019). Coupling genome-wide transcriptomics and developmental toxicity profiles in zebrafish to characterize polycyclic aromatic hydrocarbon (PAH) hazard. Int. J. Mol. Sci. 20:2570. doi: 10.3390/ijms2010 2570

Shen, M. C., Zhang, Y. X., Zhu, Y., Song, B., Zeng, G. M., Hu, D. F., et al. (2019). Recent advances in toxicological research of nanoplastics in the environment: a review. Environ. Pollut. 252, 511-521. doi: 10.1016/j.envpol.2019.05.102

Shiu, R., Vazquez, C. I., Tsay, Y., Torres, G. V., Chen, C., Santschi, P. H., et al. (2020). Nano-plastics induce aquatic particulate organic matter (microgels) formation. Sci. Total Environ. 706:135681. doi: 10.1016/j.scitotenv.2019.135681

Simon-Sánchez, L., Grelaud, M., Garcia-Orellana, J., and Ziveri, P. (2019). River Deltas as hotspots of microplastic accumulation: the case study of the Ebro River (NW Mediterranean). Sci. Total Environ. 687, 1186-1196. doi: 10.1016/j. scitotenv.2019.06.168

Sokmen, T. O., Sulukan, E., Turkoglu, M., Baran, A., Ozkaraca, M., and Ceyhun, S. B. (2019). Polystyrene nanoplastics $(20 \mathrm{~nm})$ are able to bioaccumulate and cause oxidative DNA damages in the brain tissue of zebrafish embryo (Danio rerio). Neurotoxicology 77, 51-59. doi: 10.1016/j.neuro.2019.12.010

Sokolova, I. (2018). Mitochondrial adaptations to variable environments and their role in animals'. Stress Tolerance. Integrat. Comp. Biol. 58, 519-531. doi: 10. 1093/icb/icy017

Stackley, K. D., Beeson, C. C., Rahn, J. J., and Chan, S. S. L. (2011). Bioenergetic profiling of zebrafish embryonic development. PLoS One 6:e25652. doi: 10. 1371/journal.pone.0025652

Stout, S. A., Payne, J. R., Emsbo-Mattingly, S. D., and Baker, G. (2016). Weathering of field-collected floating and stranded macondo oils during and shortly after the deepwater horizon oil spill. Mar. Pollut. Bull. 105, 7-22. doi: 10.1016/j. marpolbul.2016.02.044

Straub, L., Strobl, V., and Neumann, P. (2020). The need for an evolutionary approach to ecotoxicology. Nat. Ecol. Evol. 4:895.

Trevisan, R., Voy, C., Chen, S. X., and Di Giulio, R. T. (2019). Nanoplastics decrease the toxicity of a complex PAH mixture but impair mitochondrial energy production in developing zebrafish. Environ. Sci. Technol. 53, 8405-8415. doi: 10.1021 /acs.est.9b02003 
Williams, S. Y., and Renquist, B. J. (2016). High throughput Danio rerio energy expenditure assay. J. Vis. Exp. 2016:e53297.

Yu, F., Yang, C. F., Zhu, Z. L., Bai, X. T., and Ma, J. (2019). Adsorption behavior of organic pollutants and metals on micro/nanoplastics in the aquatic environment. Sci. Total Environ. 694:133643. doi: 10.1016/j.scitotenv.2019. 133643

Zhang, J. L., Souders, C. L. II, Denslow, N. D., and Martyniuk, C. J. (2017). Quercetin, a natural product supplement, impairs mitochondrial bioenergetics and locomotor behavior in larval zebrafish (Danio rerio). Toxicol. Appl. Pharmacol. 327, 30-38. doi: 10.1016/j.taap.2017.04.024
Conflict of Interest: The authors declare that the research was conducted in the absence of any commercial or financial relationships that could be construed as a potential conflict of interest.

Copyright $\odot 2020$ Trevisan, Uzochukwu and Di Giulio. This is an open-access article distributed under the terms of the Creative Commons Attribution License (CC BY). The use, distribution or reproduction in other forums is permitted, provided the original author(s) and the copyright owner(s) are credited and that the original publication in this journal is cited, in accordance with accepted academic practice. No use, distribution or reproduction is permitted which does not comply with these terms. 